\title{
Three stimuli for visual motion perception compared
}

\author{
HANS WALLACH \\ Swarthmore College, Swarthmore, Pennsylvania \\ ANN O'LEARY \\ Stanford University, Stanford, California \\ and \\ MARGARET L. McMAHON
Swarthmore College, Swarthmore, Pennsylvania
}

\begin{abstract}
Two arrangements yielding induced motion were used to explore the relative effectiveness of three stimulus conditions known to produce perception of motion-namely, image displacement, ocular pursuit, and object-relative displacement. In these arrangements, object-relative displacement, which resulted in induced motion, was in conflict either with ocular pursuit or with image displacement. The outcomes of these conflicts were determined by measuring the extent of induced motion. Image displacement proved more effective in competing with objectrelative displacement than did ocular pursuit, which in one arrangement yielded to object-relative displacement entirely. The same pattern of results was obtained both with the usual arrangement of the moving-center type and with a stationary-center display.
\end{abstract}

There are two distal conditions of stimulation by which the motion of a visual object is given: (1) subjectrelative, in which an object is displaced relative to the observer, and (2) object-relative, in which a moving object is displaced relative to other objects in the environment. Subject-relative displacement can produce two proximal stimulus conditions: cues from ocular pursuit when the eyes track the object, and retinal displacement of the image of the moving object when the eyes rest on a stationary point. Objectrelative displacement leads to a changing configuration in the part of the visual field where the motion occurs. In the experiments reported here, the effectiveness of these three stimulus conditions was compared.

When an object moves in a structured environment and its motion is given either by ocular pursuit or by image displacement, there will be configurational change also. Thus, as a stimulus for motion, configurational change duplicates the subject-relative stimulus conditions and carries no independent information, and this suggests the possibility that it is learned. The learning may take place when perceived motion caused by subject-relative displacement occurs in the presence of configurational change. Stimulus substitution will establish a connection between con-

\footnotetext{
This work was supported by Grant 11089 from the National Institute of Mental Health to Swarthmore College, Hans Wallach, principal investigator. We are grateful to Martha Teghtsoonian for her help in writing this report.
}

figurational change and perceived motion, making configurational change a stimulus for perceived motion of the moving object. Wallach, Bacon, and Schulman (1978) gave support to this view by showing that the quantitative relation between configurational change and the perceived motion in which it results can be rapidly altered by an adaptation procedure in which configurational change is given simultaneously with subject-relative stimulation denoting immobility of the moving object.

By its very nature, object-relative displacement is relative. When, in an otherwise homogeneous visual field, one object moves so that it approaches the other, the diminished distance between the objects is given object-relatively, but which one of the objects really moves is given subject-relatively. When the motion velocity is made to be below the subjectrelative motion threshold while configurational change remains effective-a condition that can be shown to exist-the relative nature of object-relative displacement can actually be observed. Either one of the two objects may be seen to move or both may appear to move toward each other (Duncker, 1929; Mack, Fisher, \& Fendrich, 1975). Configurational change operates very differently when one object is seen surrounded by a number of objects or against a patterned background. When here the actual motion is made to be below the subject-relative motion threshold while configurational change remains effective, it is always the single object that is seen to move and the surround is perceived as stationary. 
This rule, which was formulated by Duncker (1929), applies also when the motion is made faster and is given subject-relatively too. When the surrounded object actually moves, its motion is now given twice, subject-relatively and by configurational change. But if the object is stationary and the surround moves, the object is still seen to move due to configurational change, and the surround is seen to move because its motion is given subject-relatively. This condition causes perceived motion of a stationary object, called induced motion. It occurs only rarely under natural circumstances, but it plays a large role in research on motion.

Duncker's rule has its origin in the same learning process that presumably makes configurational change a stimulus for visual motion. If configurational change becomes a stimulus for motion perce, stion, because most moving objects that are encountered undergo displacement relative to the stationary environment while they are at the same time perceived to move due to subject-relative stimulation, part of what is here learned is that it is the surrounded object that is moving and that the surround is stationary. The perceived motion that results from configurational change imitates, so to speak, what is perceived under the most usual objective condition, namely, an object moving in a stationary surround.

When motion velocity is below the subject-relative motion threshold and only configurational change operates, induced motion reveals its true nature purely. Then the relative displacement results in motion of the surrounded object and immobility of the surround. Configurational change functions this way also when the motion of the surround is above the subject-relative threshold. Then, however, motion of the surround is seen also, but it results from the subject-relative stimulation that simultaneously operates. Induced motion is of special interest because the perceived motion of the stationary object can result only from configurational change.

In the usual case of induced motion, where a stationary object is surrounded by an array in translatory motion, the true state of this object is given by two subject-relative stimulus conditions, absence of ocular pursuit and immobility of the object's retinal image. But consider the case in which the surrounded object is itself moving. Then only one subject-relative stimulus is involved; either ocular pursuit or image displacement represents the object's motion. The displacement of the object relative to a moving surround, however, is here complex; it is the resultant of two component displacements, one due to the motion of the object and the other due to the motion of the surround. If, for instance, a dot moves vertically upward while the pattern that forms its background moves to the right, the displacement of the dot relative to the pattern is oblique, from the lower right to the upper left. Subjects see the dot move in such an oblique direction. It is interesting that this induced motion always takes place. This is not so when the surrounded object is stationary. Then a few subjects can always be found who report it as stationary. In their cases, subject-relative stimulation seems to prevail.

A special condition results when a dot is seen against a background that consists of endless parallel vertical lines moving horizontally. Their motion causes a peculiar kind of relative displacement of the dot. It has no specific direction, and only a horizontal displacement component is defined. This is so because endless straight lines cannot transmit to the eyes a particular motion direction. It would, for instance, be impossible to see whether a vertical line moves horizontally or obliquely. A vertical line can transmit to the eye only two different motion components, one to the right and the other to the left, no matter how the lines actually move. ${ }^{1}$ What is true of the lines is true also of relative displacement between the dot and the lines, which therefore defines for the dot only a displacement with a horizontal component. Thus, any motion of the dot that has as a component a displacement to the left is compatible with a motion of the line pattern to the right. As a consequence, the displacement between a dot and the line pattern can cause motion of the dot in any direction that has a horizontal component. For a particular motion direction to result, subject-relative information that determines the vertical component of the particular motion direction is needed. Thus, if the dot is stationary, that is, if its vertical component is zero, its induced motion will be horizontal, and if the dot moves vertically, its induced motion will be oblique, the resultant of the horizontal object-relative component and of its subject-relatively given objective vertical motion.

This special arrangement provides a dimensional separation of different motion stimuli. A displacement of the line pattern provides object-relative displacement of the dot in the horizontal dimension and, at the same time, a vertical objective motion of the dot is given only subject-relatively. The oblique motion that is here seen results from different motion processes, one caused by one of the subject-relative stimuli and the other by configurational change. That the two processes combine into a unitary perceived motion is a notable finding.

Inherent in all conditions that produce induced motion is a cue conflict. When the surrounded object is stationary, the object-relative displacement between it and the surround tends to make it move and the subject-relative stimulation, if fully effective, would keep it motionless. The extent of induced motion seen compared with the extent of the motion of the surround measures the relative effectiveness of the two stimulation conditions. A corresponding cue 
conflict exists when the surrounded object has a motion of its own, for instance, at right angles to a horizontal motion of the environment. The conflict is here between its oblique object-relative displacement and its vertical subject-relative displacement. The same conflict occurs in our special arrangement, where the surrounding pattern consists of vertical lines. The conflict is here between the oblique motion that, as we have shown, results as a combination of two motion processes and the subject-relatively given vertical motion of the dot. The slope angle of the perceived motion that is the outcome of this cue conflict depends on the relative effectiveness of the relative displacement between the dot and the lines, which imparts to the oblique combination motion its horizontal component, and the subject-relative stimulation, which gives the dot's motion as vertical. In the case in which the simultaneous motions, the horizontal one of the line pattern and the vertical one of the dot, are equal in extent, the slope angle of the perceived motion should be $45 \mathrm{deg}$ when the objectrelative displacement is fully effective and $0 \mathrm{deg}$, that is, vertical, when the stimulation representing the subject-relative displacement prevails. A partial yielding of subject-relative stimulation to objectrelative stimulation would result in a motion path of lesser tilt, anywhere between 0 and $45 \mathrm{deg}$. The particular deviation from verticality of the perceived motion direction represents the degree of this yielding and is a measure of the greater effectiveness of configurational change as a stimulus for motion perception.

The measured deviation from verticality can also be used to compare the effectiveness of the two subject-relative conditions of stimulation, ocular pursuit and retinal image displacement. The vertical motion of the dot can be tracked or it can be given as image displacement. The motion directions then perceived reflect the effectiveness of the two stimulus conditions when they are in conflict with the same object-relative displacement condition. A difference in the outcome of the two cue conflicts represents the difference in the effectiveness of ocular pursuit and of image displacement when they represent the same objective motion.

Measuring the deviation from verticality when the dot motion is given as image displacement requires that the subject fixate a stationary mark. But the mere presence of a stationary mark may alter the way the vertical motion of the dot is given; the mark may serve as a landmark for the dot's vertical motion, whether the motion is given by ocular pursuit or by image displacement. In our first experiment, we measured the apparent slope of the dot's motion under two conditions, when a stationary mark was fixated and when the subject tracked the moving dot in the presence of the stationary mark.

\section{EXPERIMENT 1}

\section{Method}

Subjects. Sixteen subjects, paid undergraduates, served in this experiment.

Apparatus. A translucent screen, $80 \mathrm{~cm}$ tall and $50 \mathrm{~cm}$ wide, could be made to shift left and right in reciprocating simple harmonic motion. The screen pattern consisted of evenly spaced vertical lines $2.5 \mathrm{~cm}$ apart. A small projector fashioned from a plastic tube and a single lens was mounted so that it could be tilted up and down to provide the vertical motion of a dot of $5-\mathrm{mm}$ diameter that it projected on the screen from the rear. The movement of the projector was coupled to the movement of the screen so that they reversed motion direction at the same time. ${ }^{2}$ The length of the motions of the dot and of the screen were equal and amounted to $15 \mathrm{~cm}$. One excursion always took $3 \mathrm{sec}$. A stationary mark $3 \mathrm{~mm}$ across was also projected on the screen; it was located near the vertical path of the moving dot $1.8 \mathrm{~cm}$ to its left and at a level $5 \mathrm{~cm}$ below its upper end. A test rod that was attached perpendicularly to a horizontal shaft and could be given any desired orientation in a vertical plane was located to the side of the moving screen, facing the subject, and at his or her eye level.

Procedure. There were two observation conditions; the subjects were instructed either to fixate the stationary mark or to track the moving dot. To give estimates of the apparent tilt of the path of the moving dot, the subjects observed the motion of the dot for two full cycles of the screen motion and then turned toward the test rod and adjusted its tilt to match the tilt of the dot's motion. Four such estimates were given under each observation condition, with the starting position of the rod alternately horizontal or vertical. The average of the four rod settins became the subject's estimation score. Half the subjects gave their estimates first under the fixation condition, and the other half gave theirs under the tracking condition. The distance between the subject's eyes and the screen was $40 \mathrm{~cm}$.

\section{Results}

In the fixation condition, the mean tilt estimate was $18.9 \mathrm{deg}$ and was significantly different from 0 deg $[t(15)=8.76, p<.001]$. In the pursuit condition, when the subject tracked the motion of the dot in the presence of the fixation mark, a mean tilt estimate of $27.7 \mathrm{deg}$ was obtained, which was significantly different from the mean in the fixation condition $[t(15)=4.72, p<.001]$.

The mere presence of the stationary mark in the pursuit condition had a very strong effect, as becomes evident when we compare the mean tilt estimate of $27.7 \mathrm{deg}$ with one obtained by Wallach, Bacon, and Schulman (1978) in the absence of a stationary mark but otherwise under identical conditions. With 25 subjects contributing, they obtained a mean of 43.9 deg with a standard deviation of $8.5 \mathrm{deg}$, a result that showed dominance of objectrelative displacement over ocular pursuit.

\section{EXPERIMENT 2}

We repeated Experiment 1 with two changes. First, there was a third observation condition in which the subject tracked the moving dot but the stationary mark was removed. Second, the distances between the vertical lines on the moving screen were 
made smaller by being doubled in number; the lines were now $1.25 \mathrm{~cm}$ apart.

Twelve subjects served in the pursuit condition without the stationary mark and in the image displacement condition. Half made the tilt estimates in this sequence, and the other six made them in the reverse order. Another group of 14 subjects served in the pursuit condition with the stationary mark present.

The results showed the same pattern as the one just reported. In the pursuit condition without the stationary mark, the mean tilt estimate was $45.4 \mathrm{deg}$. It was $31.8 \mathrm{deg}$ when the stationary mark was present and $23.2 \mathrm{deg}$ when the motion of the dot was given as image displacement. The last two mean tilt estimates were significantly different from each other $[t(24)=$ 2.16, p $<.025]$.

\section{EXPERIMENT 3}

One aspect of these results gave us concern. Adding a stationary mark to the condition in which the vertically moving dot is tracked caused the major change in the apparent tilt of its motion path. In the earlier experiments, adding the mark changed the mean tilt estimates from $43.9 \mathrm{deg}$ to $27.7 \mathrm{deg}$ and fixating the stationary mark caused a further change to 18.9 deg; the corresponding means in Experiment 2 were $45.4,31.8$, and 23.2 deg. We considered the possibility that adding the stationary mark in the pursuit condition was so effective because it was the only stationary object in the array that otherwise consisted of moving objects, the dot and the screen pattern. We therefore arranged a further observation condition in which the added mark underwent a small motion of its own but remained a landmark for the vertical motion of the dot. The mark was made to move horizontally back and forth at twice the rate of the reciprocating motions of the dot and the screen, over a distance of $5 \mathrm{~cm}$, which was one-third of the extent of the excursions of the dot and the screen. Otherwise, the presentation was the same as in the pursuit conditions of Experiment 2. A different group of 13 subjects each gave a set of four tilt estimates.

The result was that the moving mark had little effect on the perceived tilt of the tracked dot. The mean tilt estimate of the apparent motion of the dot was $42.4 \mathrm{deg}$, hardly different from the mean of 45.4 deg that had been obtained without the added mark. The mean tilt estimate of $\mathbf{4 2 . 4}$ deg was significantly different $[t(25)=2.80, p<.01]$ from the one of $31.8 \mathrm{deg}$ that was obtained in Experiment 2, when the added mark was stationary. Only when the additional mark was stationary did it have a sizable effect on the perceived path of the tracked dot.

\section{EXPERIMENTS 4 AND 5}

Brosgole (1968) proposed that induced motion, instead of being caused by a displacement relative to other objects, results from a change in egocentric localization connected with the Roelofs effect. Roelofs (1935) found that in the dark the straightahead direction tended to shift away from the median plane in the direction toward the center of a limited area of stimulation that was located asymmetrically to the true straight-ahead direction. Brosgole measured, by compensation, the induced motion caused by a moving frame and found that it agreed with the change in the straight-ahead direction that was caused by the frame when it was in the extreme asymmetrical position reached during its inducing motion. For several reasons, Brosgole's explanation of induced motion as resulting from a change in egocentric localization is untenable: (1) Strong induced motion is obtained when a moving pattern replaces the frame and fills the subject's visual field; (2) whereas the Roelofs effect amounts only to a part of the asymmetrical position of a limited area of stimulation, we have obtained in the tracking condition induced motion that corresponded to the full displacement of the surrounding area; and (3) when, in the same visual field, different areas are displaced in opposite directions, the two appropriate induced motions can occur simultaneously.

Bacon, Gordon, and Schulman (Note 1) recently completed an investigation in which the effects of the motion of a luminous pattern in the dark were compared under two conditions. Either the outline of the area within which the pattern moved remained stationary and centered about the subject's median plane (aligned center display) or the pattern motion resulted from the displacement of the patterned area as a whole (moving-center display). In the latter condition, Bacon et al. obtained responses indicating an egocentric change and an induced motion that was, however, larger than the egocentric change. The aligned-center display yielded no responses indicating changes in egocentric localization, but strong induced motion was measured and thus was obviously caused by configurational change. However, Bacon et al. were unable to rule out the possibility that changes in egocentric localization contribute to induced motion in moving-center displays such as those used in Experiments 1-3. Since we wanted induced motion to result from configurational change, we redid our Experiment 2 under the aligned-center condition, in which induced motion surely results from configurational change only.

\footnotetext{
Method

Subjects. Eighteen paid undergraduates served in Experiment 4 and another 18 in Experiment 5.

Procedure. Stationary black panels concealed the vertical edges of the moving screen, and transformed it into an aligned center display. Since the panels make the moving field narrower by the extent of the screen's motion, which had before amounted to $15 \mathrm{~cm}$, they would have diminished the visual angle of the horizontal extent of the line pattern from 64 to $46 \mathrm{deg}$. To avoid this, we diminished the extent of the screen motion and of the vertical motion of the dot to $9 \mathrm{~cm}$ and diminished the observation distance
} 
Table 1

Mean Tilt Estimates and Mean Tangents of Tilt Estimates With Standard Deviations Under Three Viewing Conditions

\begin{tabular}{|c|c|c|c|c|c|c|c|c|c|}
\hline \multirow{2}{*}{$\begin{array}{c}\text { Experi- } \\
\text { ment }\end{array}$} & \multicolumn{3}{|c|}{ 'Tracking } & \multicolumn{3}{|c|}{ Гracking With Mark } & \multicolumn{3}{|c|}{ Fixation } \\
\hline & Degrec: & Tangent & SD & Degrecic & Tangent & SD & Degree & Tangent & SD \\
\hline 2 & 45.4 & 1.05 & .27 & 31.8 & .66 & .30 & 23.2 & .44 & .22 \\
\hline 4 & 34.3 & .71 & .28 & 17.9 & .34 & .25 & 12.1 & .22 & .15 \\
\hline 5 & 36.7 & .78 & .30 & 2010.6 & .40 & .29 & 15.1 & .28 & .14 \\
\hline
\end{tabular}

in the same proportion, namely, to $24 \mathrm{~cm}$. The distance between the panel edges that framed the moving line pattern was $33 \mathrm{~cm}$. The stationary mark was located $1.1 \mathrm{~cm}$ to the left of the dot's vertical path. In Experiment 4, the mark was $3 \mathrm{~cm}$ below the level of the upper end, and rne excursion of the screen and of the dot took $2.1 \mathrm{sec}$. In Experiment 5 , the mark was located at the level of the center of the dot's path. and une excursion took $3 \mathrm{sec}$.

In these experiments, the same subjects served in all observation conditions, which were therefore presented in six different orders. In Experiment 4, tecause we wanted to shorten the experiment. only two tilt estimates by the subject were used in scoring. In Experiment 5, each subiect was presented with this abbreviated form of the experimen: in make comparable data available, and then the same procedure was repeated. Therefore, scores that resulted from four tilt estimates were available here also.

\section{Results}

The mean tilt estimates obtained under the three observation conditions are listed in Table 1, where they can be compared with the corresponding means from Experiment 2 . The mean tangents of the estimation scores are also listed, because the tangent of the tilt angle measures its horizontal component and is therefore proportional to the effectiveness of objectrelative displacement. Since a tangent of 1 belongs to a tilt angle of $45 \mathrm{deg}$, this value represents complete effectiveness of configurational change. In comparison with the results of Experiment 2, the mean horizontal components of the tilt estimates obtained in Experiments 4 and 5 are all significantly smaller, with $t(28)$ ranging from 2.54 to 3.22 ( $p<.02$ in each case), but the pattern of differences between the results of the different conditions remains the same. As in Experiment 2, the differences between the means for the two tracking conditions were highly significant $[\mathrm{t}(17)=6.59, \mathrm{p}<.001$, and $\mathrm{t}(17)=5.80, \mathrm{p}<$ $.001]$, and the differences between tracking with the stationary mark present and the fixation conditions were also significant $[t(17)=2.51, p=.011$, and $t(17)$ $=2.45, p=.013$ ], although the differences were smaller than in Experiment 2.

\section{DISCUSSION}

The results of the tracking condition of Experiment 2 confirmed a finding implicit in the experiments of Wallach, Bacon, and Schulman (1978) namely, that the horizontal object-relative displacement that occurs between a moving dot and its surround prevails over the stimulation produced by tracking the dot's motion. This was no longer the case in Experiments 4 and 5 , in which an aligned-center display was used. There, the horizontal component of the mean tilt estimate was smaller by about one-quarter. Whether this was entirely due to the presence of the stationary panel edges that counteracted the displacement of the line pattern is an open question. The egocentric changes that are, according to Bacon et al., associated with the moving center display used in Experiment 2 may or may not contribute to induced motion. If they do, they may have been partially responsible for the larger horizontal component of the dot's motion path which we measured in Experiment 2 .

The important findings in Experiments 4 and 5 are that, in the other two viewing conditions, the horizontal components of the dot's motion path changed in about the same proportion as they did in Experiment 2. Causing the vertical motion of the dot to be given as image displacement diminished the horizontal component of the perceived motion path by $60 \%$ or more. Of the two stimulus conditions that mediate subject-relative displacement, image displacement was more effective in overcoming configurational change than was ocular pursuit. By how much it was more effective is impossible to know, because having the dot's motion given as image displacement requires a stationary mark, which by its mere presence had a strong effect on the direction of the dot's motion when it was tracked.

The large decrease in the horizontal component of the perceived motion path that occurred when the mark was added to the tracking condition took place both when the aligned-center display was used and when the center of the line pattern moved. Our Experiment 3 was meant to test whether the mark has its effect because it functions as a landmark for the vertical motion of the dot. If that were the case, giving the mark a horizontal motion of its own should not alter its effect, for its horizontal path would still serve this function. But the moving mark failed to have the effect of the stationary mark, and this result put the landmark explanation of the effect of the stationary mark in doubt. More research is needed to gain an understanding of this large effect.

\section{REFERENCES}

Bacon, J. H., Gordon, A., \& Schulman, P. H. The effect of two types of induced motion displays on perceived location of the induced target. Perception \& Psychophysics, in press. 
Brosgone, L. An analysis of induced motion. Acta Psychologica, $1968,28,1.44$.

Duncker, K. Über induzierte Bewegung. Psychologische Forschung, 1929, 12, 180-259.

Mack, A., Fisher, C. B., \& Fendrich, R. A re-examination of two-point induced movements. Perception \& Psychophysics, $1975,17,273-276$.

Roesofs, C. O. Optische Localisation. Archive der Augenheilkunde, 1935, 109, 395-415.

WALlach, H. On perception. New York: Quadrangle/The New York Times Book Co., 1976.

Wallach, H., Bacon, J., \& Schulman, P. Adaptation in mo- tion perception: Alteration of induced motion. Perception \& Psychophysics, 1978, 24, 509-514.

\section{NOTES}

1. A more detailed explanation of the motion of endless lines is given in Wallach (1976, pp. 202-203).

2. For a more detailed description of this device, see Wallach, Bacon, and Schulman (1978).

(Manuscript accepted for publication April 7, 1982.) 